\title{
Severity Testing: A Primer
}

Jose D. Perezgonzalez

Massey University (New Zealand)

Probability theory and its offspring Statistics have a combined long history going as far back as the XVII Century (Gigerenzer et al., 1989). The interrelationships and discrepancies between Fortuna and Scientia are nowadays mirrored in our current understanding of, and approach to, both probability and statistics. The latter, for example, can be categorized into different bins, which automatically imparts particular flavours to such categories. We often talk of descriptive statistics and of inferential statistics; of hypothesis testing and of estimation; of different testing approaches such as those of Fisher, Neyman-Pearson, Bayes, and Jeffreys; and even of estimation approaches being different if conceptualized from a frequentist perspective or from a Bayesian one. The particularity categories have is that they not only help increase identification and internal consistency within categories, but also force artificial boundaries and exaggerated differences between categories (Travis \& Aronson, 2018). As Mayo (2018) put it in recently, the present state is one of statistics wars, namely between the frequentist and the Bayesian practitioners. And we could safely assume not just practitioners, as the categorizations, thus the warring, may also extend to the philosophical underpinnings of the statistical approaches involved, as we also have to contend with whether we seek confirmation of our theories (Carnap, 1962) or their falsification (Popper, 1962), and even whether the latter aligns with a Bayesian approach (e.g., Gelman \& Shalizi, 2013) or a frequentist one (Mayo, 2018) .

In the present article we shall not concern ourselves with wars, whether statistical or philosophical. Instead, we shall work on a philosophical concept being put forth by Mayo in the last two decades (e.g., Mayo, 1983, 1991, 1996; Mayo \& Spanos, 2006, 2010), more recently so with her book Statistical Inference as Severe Testing (Mayo, 2018). Severity testing stands for a procedure a researcher can avail of to falsify (frequentist) hypotheses; yet it may spill beyond that bin to become a broader philosophical approach that can serve to also falsify (Bayesian) models (Gelman \& Shalizi, 2013), even entire theories. The article I present here is going to be a primer on Mayo's severity concept in the frequentist realm. However, I sympathise with Gelman and reckon the unstated goal is to advance such primer as a step towards using severity in line with Gelman's ideas and further, including Taleb's own use of falsification as a tool to get more acquainted with what our models and theories prevents us from learning, such as about extreme events and Black Swans (e.g., Taleb, 2005, 2010).

An important aspect of Mayo's severity construct is that it is not just about statistics (otherwise, it all falls back onto mere significance testing and confidence intervals; Perezgonzalez et al., 2019). Indeed, severity testing extends to the entire procedure of research engagement, what Mayo broadly calls 'auditing'.

It is the entire procedure which needs to be stringent: audited..., multi-tested..., piecemeal testing to resolve instantiations of Duhem's problem..., formal and informal scrutiny..., etc. The aim is to achieve lift-off... via an argument from coincidence... rather than because of frequentist performance in the long run (Perezgonzalez et al., 2019, p. 2). 
Although we shall not delve in auditing per se, the forthcoming arguments rest on the assumption that such auditing has been carried out and that the entire procedure is stringent: only then we may defend an argument of statistical severity.

I have structured the article as a piecemeal, step-by-step approach to severity testing. I start with severity assessments of point nil hypotheses-these are not really of much use in current statistical inference but provide a nice introduction to the severity construct and its assessment. Section 1 delves in the pre and post assessment of severity in the case of rejection of point nil hypotheses, and Section 2 continues similar severity assessments in the case of retention of point nil hypotheses. Section 3 expands the previous assessments to the more commonly used null hypotheses, with a focus on warranted and unwarranted differences. Section 4 further expands severity assessments to the procedure of confidence intervals. Finally, in Section 5 I propose a new contribution to the assessment of severity using confidence interval in lieu of significance tests.

\section{Severity assessment in the case of rejection of the null hypothesis}

A test used to falsify a statistical null hypothesis is the typical set up of a test of significance à la Fisher (e.g., 1954). The test uses a standardized model suitable to the data at hand [e.g., $\mathrm{N}(\mu, \sigma)]$, is oftentimes informed by statistics from the sample rather than by population parameters, centres the model on a null hypothesis [i.e., $\mathrm{N}(0, \sigma)$ ], and decides on a location at the tail of that model that will serve as the level of significance for the test-the latter may be done formally or informally, a priori or post hoc, as a probability or as a critical value. The model thus serves to ascertain the tail probability of the data within that model, and the tail region serves to identify statistically significant results from which the following inference is possible: the null hypothesis has been falsified.

The technology of tests of significance is not just restricted to Fisher's approach, as we can see the same technology underlying a Neyman-Pearson's (e.g. 1933) test of acceptance (namely because the mathematical advances of contemplating an alternative hypothesis, beta, and power have no meaningful bearing on the test upon the occurrence of a significant result, except, perhaps, in quality control, when such a significant result can be formally interpreted as 'Accept $\mathrm{H}_{1}$ ', a truly fixed alternative hypothesis). We can also see it underlying confidence intervals (Neyman, 1935), especially when these are used to test whether ' 0 ', the nil hypothesis, falls outside the interval and is, therefore, statistically significant.

For maximum clarity, in this section let's consider the null model as a nil mode. Let's use ' $\mathrm{H}_{0}$ ' as shorthand for what is commonly referred to as the null hypothesis in the particular case of the nil model, whereby the effect size in question is exactly ' $O$ ' [i.e., $N(0, \sigma)$ ]. Subsequently, ' $\mathrm{noH}_{0}$ ' refers to all instances-whether formal or informal, logically correct or incorrect-of rejecting the null hypothesis $\mathrm{H}_{0}$ (Fisher's approach), rejecting the main hypothesis under test $\mathrm{H}_{M}$ (Neyman-Pearson's approach, whereby $\mathrm{H}_{M}=\mathrm{H}_{0}=0$ ), and accepting the alternative hypothesis $\mathrm{H}_{1}$ [under Neyman-Pearson's approach, whereby $\mathrm{H}_{1}$ is the precise alternative used to control the Type II error and, thus, to set up power; e.g. $N(3, \sigma)]$; it equally covers the case when ' 0 ' falls outside the confidence interval (Neyman's approach).

Two severity assessments are possible in the case of rejection of a nil hypothesis: one before carrying out the test; a second one post hoc. 


\subsection{Severity pre-assessment of the test posed by the nil model ( $S E V_{\text {HO }}$ )}

The a priori severity of the test posed by a nil hypothesis is determined by its one-tailed sensitivity (i.e., 1 - sig, $1-\alpha$ ). Indeed, a typical test with a conventional level of significance set at $5 \%^{1}$ already poses quite a big hurdle to the rejection of the nil hypothesis, as it will not reject the hypothesis whenever the observed result is equal to any of the effects found in the $95 \%$ probability density function below such significance level. Said otherwise, this is a test in favour of, or advantaging the retention of, $\mathrm{H}_{0}$; that is, it is a test against the rejection of $\mathrm{H}_{0}$. Thus,

$$
\text { a priori } \mathrm{SEV}_{\mathrm{HO}}=1-\text { sig }
$$

We shall read (1) as the (a priori) severity of the test favouring the retention of $\mathrm{H}_{0}$ (i.e., its severity against noHo). Mayo's original full exposition requires the understanding that it is the severity with which the rejection of $\mathrm{H}_{0}$ (claim noH $\mathrm{H}_{0}$ ) passes severe test $\mathrm{T}$ with data $\mathrm{x}_{0}$ [i.e., SEV (test $\mathrm{T}$, outcome $\mathrm{x}_{0}$, claim $\mathrm{noH}_{0}$ )]. We may, however, rephrase Mayo's exposition to make it inherently falsificationist; hence, it is the severity with which the retention of $\mathrm{H}_{0}$ (claim $\mathrm{H}_{0}$ ) fails severe test $T$ with data $x_{0}$ [i.e., SEV (test $T$, outcome $x_{0}$, claim $\mathrm{H}_{0}$ )]. (We shall assume the full formulation as underlying this and forthcoming formulas in the article.)

From (1) we may rest on the assurance that the nil hypothesis has failed-i.e., that the rejection of the nil hypothesis has passed-as severe a test as the one set a priori (e.g., a test with a priori SEV $=95 \%$; a.k.a., a test with $95 \%$ sensitivity). However, such assurance carries little learning derived from our research data. The most we can learn is that our results are statistically significant and, in so being, that the hypothesis has failed a severe test (whose degree of severity we already knew beforehand!). Therefore, in order to learn more from the data at hand we need to go beyond mere rejection of the nil hypothesis and into looking at significance post data.

\subsection{Severity post assessment of noHo}

In the post hoc severity of a test based on the observed result, Mayo coincides with Fisher in ascertaining that the learning that is possible is tied to the probability of the observed data within the original model under test: namely, the $p$-value actually obtained. This post-data assessment allows us to gain valuable information regarding the actual severity of the test that the nil hypothesis has failed (Mayo would put it as the severity with which the alternative hypothesis- $M_{1} \geq M_{0}$-has passed). Thus,

$$
\mathrm{SEV}_{\text {HO }}=1-\mathrm{p}
$$

In contrast to (1), we can substantiate a more specific inference with (2). For example, with a test that results in $p=0.01$, we can learn that, in the case at hand, the nil hypothesis has failed a test with as large a severity as $99 \%$. Thus, the inference that the nil hypothesis has failed a severe test is warranted more strongly with assertion (2) than with assertion (1),

\footnotetext{
${ }^{1}$ In order to improve readability, I am going to be "fast and frugal" with threshold notations for significance and power. Thus, I will use the ' $=$ ' sign in all formulations and arguments, instead of shifting between ' $=$ 'and ' $\leq$ 'or ' $\geq$ ' (unless otherwise specified). I trust the reader will be weary enough to adjust a hypothetical result with enough decimal points to make it just into a significant or non-significant result, depending on context.
} 
even if the latter was sustained by a test with an already satisfactorily high severity to start with.

Another way of looking at it-which makes Mayo's severity conceptually different from Fisher's use of the $p$-value to substantiate evidence against the nil hypothesis-is via the following counterfactual: Had the a priori severity of the test been set at the $1 \%$ level of significance (i.e., a priori $\mathrm{SEV}_{\mathrm{HO}}=99 \%$ ), the nil hypothesis would still have failed this more stringent test with the data at hand.

\section{Severity assessment in the case of non-rejection of the nil hypothesis} The main technological innovation that Neyman and Pearson (1933) brought to Fisher's test of significance was to consider a precise alternative hypothesis to provide more control over the subsequent inference, namely in regards to the possible acceptance of the nil hypothesis (see also Perezgonzalez, 2015). The mathematical advances that Neyman and Pearson incorporated to tests-specific alternative hypothesis, beta, power-allow for making an inference regarding $\mathrm{H}_{0}$ whenever we obtain a non-significant result and the a priori power of the test was high. In this case, the severity assessment of the test is determined by the alternative hypothesis, thus why we need a specific alternative $\left[\mathrm{H}_{1}\right.$; e.g. $\left.\mathrm{N}(3, \sigma)\right]$ to the nil hypothesis. Notwithstanding this, the test proper, as well as the subsequent inference, is still carried out on $\mathrm{H}_{0}$ rather than $\mathrm{H}_{1}$ (i.e., this is a test against, thus favouring the rejection of, $\mathrm{H}_{0}$ ).

Therefore, in the case of non-significant results, we also have at our disposal a set of severity assessments mirroring the ones we have learnt so far. Let's use ' $\mathrm{H}_{0}$ ' as shorthand for referring to philosophically correct but otherwise awkward statements such as non-rejection of the null (nil) hypothesis or failing to reject the null (nil) hypothesis. Therefore, ' $\mathrm{H}_{0}$ ' refers to all instances-whether formal or informal, correct or incorrect-of not rejecting the nil hypothesis $\mathrm{H}_{0}$ (Fisher's approach), retaining or accepting the main hypothesis under test $\mathrm{H}_{M}$ (Neyman-Pearson's approach, whereby $\mathrm{H}_{M}=\mathrm{H}_{0}=0$ ), even rejecting the precise alternative hypothesis $\mathrm{H}_{1}$. It also covers the case when 'O' falls within the confidence interval (Neyman's approach).

\subsection{Severity pre-assessment of the test posed by the alternative model (SEV noHo)}

The a priori severity of a test against the nil hypothesis is determined by the power of the test (i.e., by $1-\beta$, which is one-tailed). For example, a test with $95 \%$ power poses the same big hurdle against the retention of the nil hypothesis as the hurdle that a test with $95 \%$ sensitivity poses to the rejection of such nil hypothesis. Thus,

$$
\text { a priori } \mathrm{SEV}_{\mathrm{nOHO}}=1-\beta
$$

From (3) we may rest on the assurance that the nil hypothesis has passed as severe a test as the one set a priori (e.g., a test with a priori SEV $=80 \%$; a.k.a., a test with $80 \%$ power). And yet, there is little learning from our data other than to assert that the result was statistically non-significant. In order to learn more from the data at hand we need to go beyond mere "retention" of the nil hypothesis and into looking at power post data. 


\subsection{Severity post assessment of $\mathrm{H}_{0}$}

In the post assessment of severity based on the observed result, Mayo coincides with Cohen (1988) in ascertaining that the learning which is possible requires calculating the post hoc power of the observed result. This assessment post data allows us to gain valuable information regarding the actual severity of the (alternative model) test that the nil hypothesis has passed. Thus,

$$
\mathrm{SEV}_{\text {noHO }}=1-\beta_{\text {post }}
$$

In contrast to (3), we can now substantiate a more specific inference with (4). For example, with a test that returns $1-\beta_{\text {post }}=0.90$, we can learn that, in the case at hand, the nil hypothesis has passed a test with as large a severity as $90 \%$. Again, here we can ascertain that the inference that the nil hypothesis has passed the test is warranted more strongly with assertion (4) than with assertion (3), even if the latter was based on a test with satisfactorily high severity a priori.

The counterfactual way of looking at $\mathrm{SEV}_{\text {noHo }}$ is the following: Had the a priori severity of the test been set at $90 \%$ power, the nil hypothesis would still have passed that more stringent test with the data at hand.

\section{Unwarranted differences versus point hypotheses}

Despite Mayo's incursions into post data severity assessments, the knowledge we may gain from simply assessing point hypotheses is both limited and already somewhat put forth by others (i.e., Fisher, Neyman and Pearson, Cohen, etc.). These incursions may certainly provide a different interpretation for significance and may justify the post hoc assessment of power, but those are seldom novel contributions. The real contribution of Mayo's philosophy can be observed when we take the counterfactuals we discussed earlier and turn them on their heads, as we shall see.

In the following discussion, I will switch from referring to a point null hypothesis $\left(\mathrm{H}_{0}\right)$ to referring to a main hypothesis $\left(H_{M}\right)$ which still uses a null model for testing but is not necessarily bound to a nil one either philosophically or in practice (e.g., Mayo, 1983; also the idea behind equivalence tests, e.g., Lakens, 2017).

\subsection{Warranted differences upon rejecting the tested hypothesis (SEV $\mathrm{HM}_{\text {M }}$ )}

The earlier presented counterfactual regarding the severity of the rejection of the nil hypothesis posed the following: Had the a priori severity of the test been set at a level of significance equal to the observed $p$-value, the hypothesis would still have failed that more stringent test with the data at hand.

Such counterfactual-and here is Mayo's clever insight-can be turned on its head to read as follows: If we were happy to test the nil hypothesis at the a priori level of significance (e.g., sig $=5 \%$ ), we should be equally happy to accept effect sizes larger than $\mu=0$ which, had they been tested under equal conditions, would have also failed such a severe test. Thus, a significant result does not only signal that the hypothesis under test $\left(\mathrm{H}_{M}: \mu=0\right)$ has failed with severity, but also allows us to gauge which other nearby hypotheses would have equally failed the same test $\left(\mathrm{H}_{M \gamma}: \mu=0+\gamma\right)$. This assessment affords inferences regarding those effect sizes whose rejection is warranted with high severity (i.e., effects that may stand for rejectable "null" hypotheses). 
Obviously, there is no need to test those other effect sizes as the one test already gives us good information regarding which other hypotheses would equally fail the test. In a nutshell, Mayo's assessments allow us to kill more than one bird with the proverbial stone.

\subsection{Warranted differences upon accepting the tested hypothesis ( $S E V_{\text {noHm }}$ )}

Equally, the counterfactual regarding the severity assessment of accepting the nil hypothesis read as follows: Had the a priori severity of the test been set at a level of power equal to post hoc power, the hypothesis would still have passed that more stringent test with the data at hand.

Such counterfactual can also be turned on its head to read as follows: If we were happy to test against the nil hypothesis at the a priori level of power (e.g., $1-\beta=80 \%$ favouring a point alternative hypothesis), we should be equally happy to accept nearby effect sizes smaller than the point alternative which, had they informed the test under equal conditions, would also have led to $H_{M}$ passing such a severe test. Thus, a non-significant result does not only signal that the hypothesis under test $\left(\mathrm{H}_{\mathrm{M}}: \mu=0\right)$ has passed with severity, but also allows us to gauge which other nearby hypotheses would have equally passed the same test $\left(\mathrm{H}_{\mathrm{Mr}}: \mu\right.$ $=0+\gamma$ ). This assessment will equally afford inferences regarding effect sizes whose retention is warranted with high severity (i.e., effects that may stand for acceptable "null" hypotheses).

$$
\operatorname{SEV}_{\text {nоHM(0+r) }} \geq 1-\beta
$$

\section{Severity and confidence intervals}

Tests and confidence intervals are but "two sides of the same testing coin" (Perezgonzalez, 2014), and Mayo has extended severity analyses to the use of confidence intervals, more extensively so in her latest book (Mayo, 2018). Indeed, a result which just clears the significance threshold in a test of significance, will also draw a similarly directional and sensitive confidence interval which just leaves $\mu=0$ out of the interval. The only difference between both is that the test is centred on $\mu=0$ while the confidence interval is centred on the observed result (i.e., in a sense, we could say that a test of significance is no more than a confidence interval centred on $\mu=0$ ).

Mayo uses different intervals to gauge warranted and unwarranted claims with differing degrees of severity, akin to what was discussed in Section 3 above. Notwithstanding this, CIs also offer a platform for visualizing such warranted and unwarranted claims with easy. Indeed, any effect sizes that fall outside of a one-tailed confidence interval in the direction of the population parameter is warranted with as much severity as the sensitivity of the CI (or larger). Thus, a one-tailed $95 \% \mathrm{CI}$ whose lower bound clears $\mu=0$ not only indicates that the nil hypothesis has failed a severe test, but also shows which other similar claims-those up to the lower bound-would also fail such a test. As happens with tests, the nominal inference is that the effect size in the population, as warranted by the confidence procedure with the current data, is not smaller than $\mu=0+\gamma$.

Perhaps a bit more cumbersome-namely, because we do not normally consider alternative point hypotheses beyond setting power estimates-the CI visualization also applies to instances when the hypothesis under test is retained. That is, a one-tailed $95 \% \mathrm{CI}$ that does 
not clear $\mu=0$ also indicates not only that the nil hypothesis has passed a severe test, but also shows which other similar claims-those up to the upper bound-have equally passed such a severe test. The nominal inference is that the effect size in the population, as warranted by the confidence procedure with the current data, is not larger than $\mu=0+\gamma$.

\section{5 - A new contribution to assessing severity}

One element of severity that plays against itself is the manner in which Mayo comes down to explain it, which seems unnecessarily cumbersome and, thus, largely confusing. In part, this is because Mayo's interest is on assessing warranted and unwarranted claims and their corresponding levels of severity. Another reason is a philosophical anchoring on NeymanPearson's philosophy of induction, which convolutes tests a bit too much. Indeed, what Mayo is attempting to do is to use Neyman-Pearson's technology for inductive purposes, which accords rather well with Fisher's own use of tests of significance for similar purposes.

Furthermore, as insinuated earlier, when looking at testing from a merely technical perspective, Fisher's tests of significance and Neyman-Pearson's tests of acceptance are the same for the case of a significant result, namely because the mathematical improvements that Neyman and Pearson brought to testing have little bearing on such case.

In contrast, we need Neyman-Pearson's tests for the case when the hypothesis under test is retained because, as also shown earlier, the severity of the test on such hypothesis (and similar claims) depends on the power of the test. And yet we need to contend either with the stiffness of the procedure, mostly appropriate for industrial or signal/noise settings (e.g., Fisher, 1955), or with the current 'laissez-faire' practice of ignoring the alternative hypothesis once set.

In the former signal-detection case, a point alternative hypothesis has a real and valued distribution alternative to the null: The task of the tester is to decide, within some error parameters, whether the observed data is better explained by one or the other point hypothesis. Again, in such case, induction as well as Mayo's work on severity are not really needed.

In the latter laissez-faire case, the alternative hypothesis is but a 'dummy' hypothesis used for setting a starting power for the test but largely ignored thereafter, with observed results assessed only under the null hypothesis (e.g., whether significant or not) and inferences argued from it as well (e.g., which claims are warranted or not in relation to the null). Such assessment and inference are not wrong, as the test is done on the null hypothesis. However, it shows that the alternative hypothesis is used no more than as a 'dummy' hypothesis (e.g., the typical specification of the alternative as merely the opposite to the null, or $\mathrm{H}_{1}: \mu_{1}>\mu_{0}$, which, for all purposes, is indistinguishable from not stating it, as Fisher did).

Finally, we cannot forget something that has also popped up earlier, that a test of significance is no more than a confidence interval centred on $\mu=0$. This also places CIs technically and inferentially closer to Fisher's approach of using simple tests of significance than to the more mathematically elaborated Neyman-Pearson's approach, if only because a confidence procedure does not use alternative hypotheses or power.

We could thus draw a handy heuristic for assessing severity based on CIs, with the only drawback of not being able to set the severity of the test to the case of retaining the null hypothesis (i.e., not being able to set up a priori power), although such drawback is more apparent than real given that the current CI procedure also dispenses with the need for setting power. 


\subsection{Severity assessment with Confidence Intervals}

With this procedure I assume both Mayo's philosophy of severity testing as well as any of her caveats. Thus, I'm assuming proper auditing, etc. This proposal, thus, only deals with the technical aspects of confidence intervals for assessing severity.

Indeed, severity assessments become much easier when using CIs in lieu of tests of significance, more so because CIs often work with the same measures used as raw scores, so there is either no need to calculate standard effect sizes in order to describe discrepancies or such calculations are common outputs in provided by statistical packages. The interpretation of results also becomes easier because of such straight reference.

Severity assessments are always one-tailed and, indeed, we could follow Mayo and ask for one-tailed CIs, then focus on the lower or upper bound depending on whether $\mu=0$ is outside the interval (i.e., a statistically significant result) or within the interval (i.e., a statistically non-significant result), respectively.

However, we could also use the two-tailed CI for a more efficient inference. In this case, the CI will double as two one-sided tests of severity, each of which will have the same severity as per (7). That is, we can use, for example, an $80 \% \mathrm{CI}$ in lieu of two one-sided tests with $90 \%$ severity.

$$
\mathrm{SEV}_{\mathrm{CI}}=1-((1-\mathrm{CI}) / 2)
$$

Such use of CIs allows us to interpret both upper and lower claims that have failed the test with severity with the observed data (i.e., all those claims outside the interval). More importantly, we do not actually need to tie ourselves into nots regarding the location of $\mu=0$ for interpretation. Indeed, this procedure actually allows us to bypass the whole conundrum of statistical significance, as the latter now becomes largely irrelevant: $\mu=0$ is no more and no less than another hypothesis in the same continuum of alternative hypotheses (e.g., some inferences may rule out $\mu=0$ with severity, as a statistically significant test would do; some others may not rule out $\mu=0$, yet they can still rule out smaller effects, say $\mu=-1$, or $\mu=-2$ ).

Before discussing further in the conclusion, however, let's detour for a moment to see whether the proposed heuristic stands to test via empirical replication of Mayo's own assessments.

\subsection{Is this correct? The empirical work}

Mayo (e.g., 1983, 2018) already hinted that, given the intimate mathematical relationship between tests of significance and confidence intervals, one-tailed CIs could also inform about severity assessments. Although she has not produced a proposal like the one here described, she does provide a multitude of test-based severity assessments which can serve to put such proposal to test. Thus, I have scouted as many of her severity assessments as possible, finding enough of them, whether in-text, graphic, or tabular form, in Mayo (1983, 2012, 2018), Mayo and Spanos (2006, 2011), and Spanos (2014).

Out of a total of 83 severity assessments collated, 81 assessments (96.4\%) were replicated using the proposed CI heuristic at the decimal levels reported in those works. The three assessments not replicated were found in an earlier work by Mayo and Spanos (2006), in the section discussing significance and severity assessments under varying samples sizes. In this section, Mayo and Spanos did not clarify which standard deviation they were using, so when 
plugging in the standard deviation they used in that article's other examples $(\sigma=2)$, the results did not replicate. However, a very similar set of assessments is found in Spanos (2014), in which only the effect size and the standard deviation differ. When substituting Spano's (2014) standard deviation $(\sigma=1)$ and rounding the sample mean to one decimal place, two of the suspected assessments replicated at the reported decimal level. Thus, if Mayo and Spanos did, indeed, used a different standard deviation when discussing sample size effect on severity in 2006 , then $98.8 \%$ of the assessments replicated perfectly at the reported decimal level ${ }^{2}$. These results, thus, support the proposed use of CIs as a good heuristic for Mayo's technology of severity assessments.

\section{Conclusion}

Mayo's severity procedure first needs to ascertain whether the test is significant or not so as to determine which tail to use for severity assessment (i.e., we may be only interested in claims that are warranted with large severity, which only occurs for claims that diverge towards the opposite tail to where the observed data is located when the data is significant, yet diverge farther away from the observed data but on the same tail when not significant). Afterwards, it needs to calculate the Z-scores for the differences between data and several alternative claims, which practically limits assessments to the normal distribution. In calculating the tail probability of the corresponding Z-scores, we finally learn the varying degrees of severity for such alternative claims.

Thus, we may learn, for example, that a significant result that rejects the main (null) claim that the population mean is equal to or smaller than $150\left[H_{M}: \mu \leq 150 ; \bar{x}=152, N(150,10), n=\right.$ 100 ], allows for alternatives inferences such as $\mu \nless 151$ with severity $=84 \%, \mu \nless 150$ with severity $=97 \%$, and $\mu \nless 149$ with severity $99.9 \%$. Equally, a non-significant result that fails to reject such main claim $(\bar{x}=151)$ allows for alternatives inferences such as $\mu \ngtr 152$ with severity $=84 \%$, and $\mu \ngtr 153$ with severity $=97 \%$ (Mayo, 2018).

A CI-based severity assessment can warrant similar inferences with ever precise coverage. For example, the same observed data $(\bar{x}=152)$ replicates the severity assessment with $\mathrm{SEV}_{\mathrm{CI} 68.27}[151,153]$, and $\mathrm{SEV}_{\mathrm{C} 195.45}[150,154]$, both indicating not only that their lower bounds clear the stated claims ( $\mu=151, \mu=150$, respectively), thus are statistically significant, but also that claims about rejecting smaller effects are warranted with high severity (84\% and $98 \%$, respectively, after converting the two-tailed CI coverage to a one-tailed one).

However, such calculations are not the real value of using the CI heuristic, as the heuristic is redundant if the only intention is to replicate Mayo's procedure. Instead, its real value is the opportunity to ask a different set of questions. Firstly, which claims are warranted with the observed data for a given level of severity? For $\bar{x}=152[N(152,10), n=10]$, the warranted claims are that, with $90 \%$ severity (one-tailed), the population mean is not smaller than 148 , or that is not larger than 156 (i.e., $\operatorname{SEV}_{\mathrm{CI80}}[147.95,156.05]$ ), and with $99 \%$ severity, that the population mean is not smaller than 144.7 (i.e., $\mathrm{SEV}_{\mathrm{CI} 98}=144.7,159.4$ ). (Notice that we may simply report one of the bounds, as appropriate, as we are reporting severity, not confidence intervals.)

Secondly, the CI heuristic opens up the opportunity to report severity using distributions other than the normal. For example, the same observed data would yield slightly wider

\footnotetext{
${ }^{2}$ Because the very similar assessments by Spanos (2014) did replicate perfectly, my suspicion is that Mayo and Spanos's assessment for $n=100$ and $d\left(x_{0}\right)=1.96$, SEV $(\mu>12.1)=0.833 ; H_{0}=12, N(12,1)$ contains an error somewhere. The result I obtained with such data was SEV $=0.832$ (without rounding $d\left(x_{0}\right)=1.96 ; \bar{x}=12.196$ ), or SEV $=0.841$ (when rounding $d\left(\mathrm{x}_{0}\right)=1.96$ to $\overline{\mathrm{x}}=12.2$ ).
} 
estimates when using the t-distribution ( $\bar{x}=152[T(152,10), n=10])$, with the associated claims that, with $90 \%$ severity, the population mean is not smaller than 147.6 (i.e., $\mathrm{SEV}_{\text {C180 }}$ $[147.6,156.4]$ ) and, with $99 \%$ severity, that it is not smaller than 143 (i.e., $\mathrm{SEV}_{\mathrm{C} 198}$ [143.1, 160.9]).

Thirdly, as indicated earlier, the CI approach allows us to bypass the need for a null hypothesis and, therefore, the whole conundrum of statistical significance (e.g., Lakens et al., 2018; Trafimow et al., 2018; Hurlbert, Levine \& Utts, 2019; Amrhein, Greenland \& McShane, 2019). For example, let's say we get a score of 151 in some measure with a sample of 100 participants, a measure which we know is about $\mu=150(\sigma=10)$ in the population at large. The $80 \%$ SEV $_{C I}[149.72,152.2]$ signals that the sample is not too far from the population's parameter; indeed, with $90 \%$ severity, we may warrant the claim that it is not much larger than about $\mu=152$ (even, perhaps, that it is not much smaller than $\mu=149.7$, either). Thus, whether the result is not significant and whether a null hypothesis is retained or cannot be rejected need not enter into our inferred claims.

Fourthly, the CI-bound severity assessment also allows for more honest interpretations of the importance of research results. By bypassing the significance statement, results that would have otherwise turned up significant with a test are now assessed in relation to the location of the lower bound of the CI interval, only. For example, let's say we are tempted to increase sample size to "ensure" a significant result with the statistic just observed $(\bar{x}=151)$. With 10 times the sample size $(n=1000)^{3}$, we may get a significant result, as the CI just clears $\mu=0$ ( $\left.\mathrm{CI}_{95}[150.38,151.62]\right)$. The severity assessment, however, even when rather liberal $\left(\mathrm{SEV}_{\mathrm{CI} 80}[150.6,151.4]\right.$; i.e. $\left.\mathrm{SEV}=90 \%\right)$, only warrants the claim that the effect size is not smaller than $\mu=150.6$, a rather trivial difference irrespective of its statistical significance.

On the other hand, such more relevant interpretations extend even to the upper bound. Let's say we run a pilot study to check the viability of a project. Even with provisional results taken from a small sample $[\bar{x}=151, N(150,10), n=10]$, a severity assessment may signal important claims that we may miss in the full study (SEV $\left.\operatorname{SI80}_{\text {II }}[146.95,155.05]\right)$. Assume we are interested in an effect size larger than $\mu=155$ as for deeming the project worthwhile. The severity assessment already indicates that we have pretty much missed such effect, even with the generous conditions of a wide interval due to this sample's large standard deviation. We can now proceed in two ways. We could carry out the full study somewhat reassured that the larger sample size of the study proper will reduce the standard deviation and produce a significant result (we may even decide to procure a larger sample than initially envisioned); in so doing we will favour statistical significance over practical importance. Or we could learn from the data at hand that the intervention may not be good enough as for guaranteeing the desired effect; therefore, the intervention would need reformulating before going into the full study (or it may need to be dropped altogether).

In summary, the use of confidence intervals for severity assessments may prove to be easier to adopt than one based on tests, with the only caveat of having to remember that the heuristic considers two one-tailed CIs, not merely a two-tailed one. Despite this, the question remains whether simply using the typical $95 \%$ CI would not suffix. The answer, of course, is, 'Yes, it would suffix', as long as we remember that the severity assessment is short of $98 \%$, thus perhaps much greater than needed in an omnibus manner. Interestingly enough, a $90 \%$ severity assessment is achieved with an $80 \% \mathrm{CI}$, an interval width which Cohen (1988) already considered as more reasonable for the behavioural (and social) sciences.

\footnotetext{
${ }^{3}$ This is just a mere counterfactual, to illustrate that obtaining a significant result gets easier the larger the sample size. Acting as above would void any severity assessment in virtue of the procedure failing its auditing.
} 
All in all, despite the potential value of the CI heuristic, the critical point to consider is that the severity assessment is not about the CI interval, which is to be interpreted not in a confirmatory manner but in a falsificationist one. What the $\mathrm{CI}$ identifies are not those claims within the interval as plausible-nor as being increasingly more plausible as they get closer to the statistic, e.g. Cummings, 2012)-but those claims outside the interval that have been falsified with the data at hand at the reported level of severity. We may even entertain the related inference that claims further away from the corresponding interval bound are ever more warranted so with an increasing degree of severity.

\section{References}

Amrhein, V., Greenland, S., \& McShane, B. (2019). Scientists rise up against statistical significance. Nature, 567, 305-307. https://doi.org/10.1038/d41586-019-00857-9

Carnap, R. (1962). Logical foundations of probability, 2nd Edn. The University of Chicago Press.

Cohen, J. (1988). Statistical power analysis for the behavioural sciences, 2nd Edn. Erlbaum.

Cumming, G. (2012). Understanding the new statistics. Routledge.

Fisher, R. A. (1954). Statistical methods for research workers, 12th Edn. Oliver and Boyd.

Fisher, R. A (1955). Statistical methods and scientific induction. Journal of the Royal Statistical Society, Series B (Methodological), 17(1), 69-78.

Gelman, A., and Shalizi, C. R. (2013). Philosophy and the practice of Bayesian statistics. British Journal of Mathematical and Statistical Psychology, 66, 8-38. https://doi.org/10.1111/j.2044-8317.2011.02037.x

Gigerenzer, G., Swijtink, Z., Porter, T., Daston, L., Beatty, J., Kruger, L. (1989). The empire of chance. Cambridge University Press.

Hurlbert, S. H., Levine, R. A., Utts, J. (2019). Coup de grâce for a tough old bull: "statistically significant" expires. The American Statistician, 73:sup1, 352-357.

https://doi.org/10.1080/00031305.2018.1543616

Lakens, D. (2017). Equivalence tests: a practical primer for $t$ tests, correlations, and metaanalyses. Social Psychological and Personality Science, 8(4), 355-362.

https://doi.org/10.1177\%2F1948550617697177

Lakens, D., Adolfi, F. G., Albers, C. J., Anvari, F., Apps, M. A. J., Argamon, S. E., Baguley, T., Becker, R. B., Benning, S. D., Bradford, D. E., Buchanan, E. M., Caldwell, A. R., Van Calster, B., Carlsson, R., Chen, S. C., Chung, B., Colling, L. J., Collins, G. S., Crook, Z., Cross, E. S., . .

Zwaan, R. A. (2018). Justify your alpha. Nature Human Behavior, 2, 168-171.

https://doi.org/10.1038/s41562-018-0311-x

Mayo, D. G. (1983). An objective theory of statistical testing. Synthese, 57(3), 297-340.

https://doi.org/10.1007/BF01064701

Mayo, D. G. (1991). Novel evidence and severe tests. Philosophy of Science, 58(4), 523-552. https://doi.org/10.1086/289639

Mayo, D. G. (1996). Error and the growth of experimental knowledge. The Chicago University Press. 
Mayo, D. G. (2012). Statistical science meets philosophy of science. Part 2: shallow versus deep explorations. RMM, 3, 71-107.

Mayo, D. G. (2018). Statistical inference as severe testing. Cambridge University Press.

Mayo, D. G., and Spanos, A. (2006). Severity testing as a basic concept in a Neyman-Pearson philosophy of induction. The British Journal for the Philosophy of Science, 57(2), 323-357. https://doi.org/10.1093/bjps/axl003

Mayo, D. G., and Spanos, A. (2011). Error statistics. In P. S. Bandyopadhyay and M. R. Forster (Eds.), Philosophy of statistics, Volume 7. Elsevier.

Mayo, D. G., and Spanos, A. (Eds.) (2010). Error and inference. Cambridge University Press.

Neyman, J. (1935). On the problem of confidence intervals. Annals of Mathematical Statistics, 6(3), 111-116. https://doi.org/10.1214/aoms/1177732585

Neyman, J., and Pearson, E. S. (1933). On the problem of the most efficient tests of statistical hypotheses. Philosophical Transactions of the Royal Society of London A: Mathematical, Physical and Engineering Sciences, 231, 289-337. https://doi.org/10.1098/rsta.1933.0009

Perezgonzalez, J. D., Pascual-Soler, M., Pascual-Llobell, J., \& Frias-Navarro, D. (2019). Book review: Statistical inference as severe testing. Frontiers in Psychology, 10. 735.

https://doi.org/10.3389/fpsyg.2019.00735

Popper, K. (1962). Conjectures and refutations. Basic Books.

Spanos, A. (2014). PHIL 6334 - Probability / Statistics Lecture Notes 5: Post-data severity evaluation. https://www.slideshare.net/jemille6/spanos-lecture-5-6334severity2014

Taleb, N. N. (2005). Fooled by randomness, 2nd Edn. Random House.

Taleb, N. N. (2010). The Black Swan, 2nd Edn. Random House.

Trafimow, D., Amrhein, V., Areshenkoff, C. N., Barrera-Causil, C. J., Beh, E. J., Bilgiç, Y. K., Bono, R., Bradley, M. T., Briggs, W. M., Cepeda-Freyre, H. A., Chaigneau, S. E., Ciocca, D. R., Correa, J. C., Cousineau, D., de Boer, M. R., Dhar, S. S., Dolgov, I., Gómez-Benito, J., Grendar, M., Grice, J. W., . . Marmolejo-Ramos, F. (2018). Manipulating the alpha level cannot cure significance testing. Frontiers in Psychology, 9:699. https://doi.org/10.3389/fpsyg.2018.00699

Travis, C., and Aronson, E. (2016). Mistakes were made (but not by me). Pinter \& Martin. 